\title{
Role of bispectral index monitoring and burst suppression in prognostication following out-of-hospital cardiac arrest: a systematic review protocol
}

\author{
Leanne Eveson ${ }^{1}$, Marcela Vizcaychipi ${ }^{2}$ and Shashank Patil ${ }^{1 *}$
}

\begin{abstract}
Background: Out-of-hospital cardiac arrest (OHCA) is associated with significant mortality or may have a poor neurological outcome. Various community-training programmes have improved practices like bystander cardiopulmonary resuscitation (CPR) and early defibrillation using automated external defibrillator (AED). Post-resuscitation care has also changed significantly in the millennium. Interventions like targeted temperature management (TTM), avoidance of hyperoxia and emergency cardiac catheterisation have given patients a chance of a better neurological outcome. Despite these timely interventions, it is still very difficult to predict neurological outcome. The European Resuscitation Council and European Society of Intensive Care Medicine (ERC-ESICM) published guidance in 2015 with a strong recommendation to delay prognostication for at least $72 \mathrm{~h}$ and with an emphasis to adapt a multimodal approach, which includes neurological examination, biomarkers, electroencephalogram (EEG) and radiological tests. These interventions not only have cost attached to them, but the unpredictability has a significant emotional impact on family members. Bispectral index (BIS) monitoring device acts on the principle of EEG and converts the waveform into an absolute number and also measures the burst suppression. We hypothesize that patients who have a low BIS value and high burst suppression within $24 \mathrm{~h}$ of presentation will have a poor neurological outcome. The primary objective of this review is to look at BIS monitor as a tool, which could help bring forward the timing of prognostication.

Methods: Electronic databases will be systematically searched for randomised controlled trials and prospective or retrospective cohort studies with no language restrictions. The search will be supplemented with grey literature searches of thesis, dissertations and hand searching of relevant journals. Two independent reviewers will screen, select and perform analysis according to the Preferred Reporting Items for Systematic Review and Meta-analysis (PRISMA) method. The selected studies will be analysed using the Grades of Recommendation, Assessment, Development and Evaluation (GRADE) system. Meta-analysis will be performed if suitable.
\end{abstract}

Discussion: This review will synthesize the evidence on the use of BIS monitors within $24 \mathrm{~h}$ of achieving return of spontaneous circulation (ROSC) and may help in early prognostication.

Systematic review registration: PROSPERO CRD 42016050224.

Keywords: Bispectral index, BIS, Burst suppression, Out-of-hospital cardiac arrest, OHCA, Prognostication

\footnotetext{
* Correspondence: shashank.patil@chelwest.nhs.uk

${ }^{1}$ Emergency Medicine, Chelsea and Westminster Hospital NHS Foundation

Trust, 369 Fulham Road, London SW10 9NH, UK

Full list of author information is available at the end of the article
} 


\section{Background}

Out-of-hospital cardiac arrest (OHCA) is associated with a high mortality. Average survival to hospital discharge for OHCA patients in England in 2013 was only $8.6 \%$ [1]. Despite advances in post-resuscitation care management, about $50 \%$ of resuscitated patients from cardiac arrest (CA) die or have a poor neurological prognosis. One of the major causes of mortality following $\mathrm{CA}$ is severe neurological damage due to post-anoxic brain injury [2]. The cost and length of stay is higher in patients with poor neurological outcome, which can be up to $£ 50,000$ per survivor [3]. There are further considerations like community care and rehabilitation, quality of life and emotional impact on the family. It is therefore essential to predict neurological outcome in this group of patients as early as possible, in order to potentially enable early withdrawal of life-saving treatment (WLST) in those patients predicted to have a poor outcome.

Cardiac arrest causes a significant pathological and clinical impact, known as 'post-cardiac arrest syndrome' [4].

The four key components of post-cardiac arrest syndrome were identified as:

I. Post-cardiac arrest brain injury;

II. Post-cardiac arrest myocardial dysfunction;

III.Systemic ischaemic / reperfusion injury;

IV.Persisting precipitating pathology

Post-resuscitation care aims to reduce this impact; it has developed and evolved significantly since 2003, following recommendations by the Advanced Life Support (ALS) task force of the International Liaison Committee on Resuscitation (ILCOR) to implement Therapeutic Hypothermia (TH) in unconscious survivors following OHCA [5]. The 2015 European Resuscitation Council (ERC) and European Society of Intensive Care Medicine (ESICM) guidelines on postresuscitation care made strong recommendations to avoid severe hyperoxia (large amounts of oxygen) for patients following CA [6-8], emergency cardiac catheterisation \pm immediate percutaneous coronary intervention (PCI) and targeted temperature management (TTM) between $32-36{ }^{\circ} \mathrm{C}$ [9].

In 2015, ERC-ESICM recommended a multimodal prognostication approach for comatose survivors following CA [10]. It was based on a robust analysis of evidence and provided a practical recommendation. Hence, it formed the basis of ERC guidelines on resuscitation published in 2015.

The key recommendations are summarised below with a great emphasis on the fact that they should be used in conjunction with each other:

1. Clinical examination
- Using bilateral pupillary and corneal reflexes at $72 \mathrm{~h}$ or more from return of spontaneous circulation (ROSC) to predict poor outcome in comatose survivors from cardiac arrest, either $\mathrm{TH}$ or non-TH treated patients;

2. Myoclonus and status myoclonus

- Using the term status myoclonus to indicate a continuous and generalised myoclonus persisting $>30 \mathrm{~min}$ in comatose survivors of $\mathrm{CA}$;

- Using the presence of a status myoclonus within $48 \mathrm{~h}$ from ROSC in combination with other predictors to predict poor outcome in comatose survivors of $\mathrm{CA}$, either $\mathrm{TH}$ or non-TH treated.

3. Bilateral absence of SSEP (somatosensory-evoked potentials) N20 wave

- Using bilateral absence of SSEP N20 wave at $\geq 72 \mathrm{~h}$ from ROSC to predict outcome in comatose survivors following CA treated with controlled temperature;

- There was suggestion to use SSEP at $\geq 24 \mathrm{~h}$ from ROSC to predict outcome in comatose survivors following CA not treated with controlled temperature.

4. Electroencephalogram (EEG)

- Absence of EEG reactivity to external stimuli, presence of burst suppression or status epilepticus at $\geq 72 \mathrm{~h}$ after ROSC to predict poor outcome in comatose survivors from CA.

\section{Biomarkers}

- There is suggestion to use high NSE (neuron-specific enolase) at 48-72 $\mathrm{h}$ from ROSC in combination with other predictors for prognosticating a poor neurological outcome in comatose survivors following CA, either TH or non-TH treated.

\section{Imaging}

- Using the presence of a marked reduction in grey matter/white matter (GW/WM) ratio or sulcal effacement on brain CT within $24 \mathrm{~h}$ after ROSC or presence of the extensive reduction in diffusion on brain MRI at 2-5 days after ROSC to predict a poor outcome in comatose survivors following CA both $\mathrm{TH}$ or non-TH treated.

The quality of evidence on which the above strategy is developed and recommended is low to very low. Golan et al. in their meta-analysis showed that only three tests 
accurately predicted poor prognosis with low false positive rates (FPR): bilateral absences of pupillary reflex more than $24 \mathrm{~h}$ after CA (FPR 2\%, confidence interval (CI) 1-6\%), bilateral absence of corneal reflex more than $24 \mathrm{~h}$ post CA (FPR 4\%, CI 1-9\%) and bilateral absence of SSEP between day 1 and 7 (FPR 3\%, CI 1-7\%). FPR were higher for a Glasgow Coma Score-Motor response (GCS-M) less than 2, unfavourable EEG patterns, myoclonic status epilepticus and elevated NSE [11].

Also, despite the recommended delayed prognostication strategy, a large single-centre study of 326 patients found that $30 \%$ of patients have delayed awakening (i.e. still in coma after TTM and sedation withdrawal) and up to $20 \%$ remained comatose at 1 week [12].

There are other modalities like bispectral index (BIS) monitor and infrared pupillometry, which are currently being trialled to predict neurological outcome [13-15]. These are non-invasive techniques and can be used in emergency or intensive care settings with a certain degree of training. We suggest, given the available low quality of evidence and recommended multimodal approach, that there is definitely a place for a new modality. This study aims to look at the available evidence to support early use of Bispectral Index and burst suppression (BR) monitoring especially in the emergency department (ED) to help predict neurological outcome.

\section{BIS monitor}

BIS monitor is the brain monitoring system for critical care developed by Covidien-Medtronic. A sensor is placed on the patient's forehead and raw electroencephalogram (EEG) data is collected. The EEG information is processed by the system and calculates a number between 0 and 100. This provides a direct measure of a patient's level of consciousness. See Table 1 for interpretation of BIS value and clinical state.

\section{BSR}

During the burst suppression phase, suppression wave (amplitude $<0.5 \mu \mathrm{V}$ ) follows burst wave (amplitude > $0.5 \mu \mathrm{V})$. The suppression ratio is expressed as a percentage and is the ratio of total duration of suppression wave to total duration of analysis [16].

Table 1 BIS value and clinical states

\begin{tabular}{ll}
\hline $\begin{array}{l}\text { BIS index } \\
\text { range }\end{array}$ & Clinical state \\
\hline 100 & Awake: responds to normal voice \\
80 & Light to moderate sedation: may respond to loud commands \\
60 & General anaesthesia \\
40 & Deep hypnotic state \\
20 & Burst suppression \\
0 & Flat-line EEG \\
\hline
\end{tabular}

\section{Primary hypothesis}

In a patient who remains comatose following ROSC after cardiac arrest, low BIS value $(<20)$ and high burst suppression ratio is a predictor of poor neurological outcome. The aim of this study is to analyse the available evidence to support early use of BIS and BSR monitoring in the ED to help predict neurological outcome.

\section{Methods}

The systematic review will be designed in accordance with the Preferred Reporting Items for Systematic Reviews and Meta-analyses (PRISMA-P statement) [17]. The systematic review protocol is provided in Additional file 1 . This protocol is registered with PROSPERO as CRD42016050224.

\section{Eligibility criteria \\ Study design}

This systematic review will include randomised controlled studies and prospective and retrospective cohort studies. A grey literature search of thesis and dissertations and hand searching of relevant journals will also be carried out. The study will include adult (age greater than or equal to 18 years) cardiac arrest patients. All arrest rhythms will be included. Studies will be included if they report the use of BIS monitoring and/or BSR monitoring and if the BIS and BSR monitoring were initiated within 24 h of ROSC. Studies will be included in which the reported outcome of interest is neurologically-intact (cerebral performance category CPC 1-2). Studies will be excluded if the outcome of interest is survival rather than performance status, if BIS or BSR monitoring was commenced during resuscitation effort or commenced later than $24 \mathrm{~h}$ following ROSC, and all case reports will be excluded.

\section{Information sources}

Studies will be identified through a systematic search of the following electronic databases: MEDLINE (Ovid), EMBASE (Ovid), CINHAL (Ebsco), Cochrane Database of Systematic Reviews (CDSR), DARE (Database of Abstracts of Reviews of Effectiveness), LILACS (Latin American and Caribbean Health Sciences Literature), International Clinical Trials Registry Platform (ICTRP), Science citation index-expanded (SCI-expanded) and Conference Proceedings Citation Index-Science (CPCI-S), as well as clinical trials, dissertations and theses. PROSPERO repository will also be searched for all active or completed systematic reviews. Searches of electronic databases will be supplemented with discussions with authors of unpublished studies, inspection of reference lists of relevant articles and hand searching of pertinent journals. As BIS was introduced in 1994, searches from 1994 onwards are considered sufficient. 


\section{Search strategy}

The search strategy was designed and conducted by an information specialist experienced in systematic reviews $\mathrm{PB}$ following the Cochrane handbook for systematic review of interventions [18]. The search includes controlled vocabulary $(\mathrm{MeSH})$ and natural language terms: Bispectral index, BIS, burst suppression, out-of-hospital cardiac arrest, OHCA and prognostication. If a study is considered partially eligible based on the abstract, we will attempt to extract the required data. If this cannot be done, we will include this in the report of the review. The final search strategy will be peer-reviewed by an independent medical information specialist using the Peer Review of Electronic Search Strategies (PRESS) checklist [19]. No language restriction will be applied. The search will be updated at the end of our review to ensure the most recent relevant articles are included. A detailed search strategy for MEDLINE and EMBASE is provided at Additional file 2.

\section{Data management}

The results of the literature search will be stored in HDAS (Healthcare Databases Advanced Search) and screened according to the study selection process. The selected studies will be analysed using the Grades of Recommendation, Assessment, Development and Evaluation (GRADE) system [20]. The data extraction template for Cochrane reviews [21] will be used to create the study eligibility form, please see Additional file 3.

\section{Study selection}

A hierarchical screening method adapted from PRISMA will be used by two reviewers, SP and LE. The reviewers will independently screen the titles and abstracts highlighted by the search strategy. Eligible studies will be selected for full text analysis. Reviewers will record reasons for exclusion of studies in a data extraction form based on the data extraction template for Cochrane reviews. Authors will not be blinded to the journal titles, authors or institutions.

The following data will be extracted from each eligible study: patient age, survival to discharge, use of therapeutic hypothermia, use of neuromuscular blockade, BIS/BSR use, BIS level and/or BSR for those with CPC $1-2$ versus $\mathrm{CPC} 3-5$, time point of BIS monitoring, sensitivity and specificity of BIS level studies and primary outcome. Study design, sample size, year of study conduct, year of study publication and country of origin will also be recorded.

If required, authors of eligible studies will be contacted for discussion. All discussions with authors will be documented. Data extracted will be collected and organised in a Microsoft Excel spreadsheet.
Risk of bias and quality assessment of individual studies All eligible studies will be assessed for risk of bias (RoB) using the RoB in non-randomised studies of interventions (ROBINS-I) tool [22] and the Cochrane RoB tool for randomised controlled trials [23]. Disagreements between reviewers will be recorded and discussed and resolved by an independent reviewer.

\section{Statistical analysis}

The aim of this review is to determine the association between patients who have a low BIS value and high BSR within $24 \mathrm{~h}$ of presentation post OHCA and neurological outcome. The results from all studies will be included in statistical analysis. Odds ratios will be used to examine the role of BIS as a prognostic factor for neurological outcome. We will calculate pooled estimates of effect of intervention, together with $p$ values and confidence intervals. Variation will be checked for between studies (heterogeneity) and we will also analyse publication bias. We assume there will be heterogeneity due to limited evidence in this field and will apply the randomeffects model [24]. The methods that will be used to test for heterogeneity are $I$-square statistics [25]. If significant heterogeneity is found, we will perform a sensitivity or sub-group analysis. Meta-regression will be used to examine the causes of any significant heterogeneity.

\section{Discussion}

OHCA is associated with significant mortality or may have a poor neurological outcome. Prognostication of OHCA survivors poses a significant challenge. The current delayed multimodal prognostication model is recommended on the basis of currently available low to very low quality of evidence. BIS monitoring is currently not widely used; however, it is relatively simple to use, easy to train operators, non-invasive, can be used in emergency and intensive care settings and multiple readings can be available at various timelines during postresuscitation care management. This review will synthesize the evidence available on the use of BIS monitors at various timelines after achieving ROSC and may help contribute as an additional modality to the current multimodal prognostication model.

\section{Additional files}

Additional file 1: PRISMA-P (Preferred Reporting Items for Systematic review and Meta-Analysis Protocols) 2015 checklist: recommended items to address in a systematic review protocol. (PDF $145 \mathrm{~kb}$ )

Additional file 2: Search Strategy on MEDLINE and EMBASE. (PDF 97 kb)

Additional file 3: Cochrane public health group data extraction and assessment template (DOCX $93 \mathrm{~kb})$ 


\section{Abbreviations}

AED: Automated external defibrillator; ALS: Advanced life support; BIS: Bispectral index; BSR: Burst suppression ratio; CDSR: Cochrane Database of Systematic Reviews; CPC: Cerebral performance category; CPClS: Conference Proceedings Citation Index-Science; CPR: Cardiopulmonary resuscitation; CT: Computed tomography; DARE: Database of Abstracts of Reviews of Effectiveness; ED: Emergency Department; EEG: Electroencephalogram; ERC: European Resuscitation Council; ERCESICM: European Resuscitation Council and European Society of Intensive Care Medicine; ESICM: European Society of Intensive Care Medicine; FPR: False positive rates; GCS-M: Glasgow Coma Score-Motor response; GM WM: Grey matter/white matter; GRADE: Grades of Recommendation, Assessment, Development and Evaluation; HDAS: Healthcare Databases Advanced Search; ICTRP: International Clinical Trials Registry Platform; ILCOR: International Liaison Committee on Resuscitation; LE: Leanne Eveson; LILACS: Latin American and Caribbean Health Sciences Literature; MeSH: Medical subject headings; MRI: Magnetic resonance imaging: MV: Marcela Vizcaychipi; NSE: Neuron-specific enolase; OHCA: Out-of-hospital cardiac arrest; PCI: Percutaneous coronary intervention; PRESS: Peer Review of Electronic Search Strategies; PRISMA: Preferred Reporting Items for Systematic Review and Meta-analysis; RoB: Risk of bias; ROBINS-I: Risk of bias in non-randomised studies of interventions; ROSC: Return of spontaneous circulation; SCI: Science citation index; SP: Shashank Patil;

SSEP: Somatosensory-evoked potentials; TH: Therapeutic hypothermia; TTM: Targeted temperature management; WLST: Withdrawal of life-saving treatment

\section{Acknowledgements}

The authors would like to acknowledge the contribution of Mr. Philip Barlow, who assisted in developing the search strategy.

\section{Funding}

Not applicable

\section{Availability of data and materials}

All data generated or analysed during this study are included in this published article and its supplementary information files.

\section{Authors' contributions}

The manuscript was prepared by LE, MV and SP. All authors read and approved the final manuscript.

Ethics approval and consent to participate

Ethics approval and consent waived.

\section{Consent for publication}

Not applicable

\section{Competing interests}

The authors declare that they have no competing interests.

\section{Publisher's Note}

Springer Nature remains neutral with regard to jurisdictional claims in published maps and institutional affiliations.

\section{Author details}

${ }^{1}$ Emergency Medicine, Chelsea and Westminster Hospital NHS Foundation Trust, 369 Fulham Road, London SW10 9NH, UK. ${ }^{2}$ Anaesthesia \& Intensive Care Medicine, Chelsea and Westminster Hospital NHS Foundation Trust, 369 Fulham Road, London SW10 9NH, UK.

Received: 9 June 2017 Accepted: 18 September 2017

\section{Published online: 25 September 2017}

\section{References}

1. Consensus paper on out-of-hospital cardiac arrest in England. Oct 2014. https://www.bhf.org.uk/-/media/files/publications/ohca-consensus-paper.pdf

2. Nolan JP. Neuroprognostication after cardiac arrest in Europe: new timings and standards. Editorial/Resuscitation. 2015;90:A4-5.

3. Petrie J, Easton S, Naik V, et al. Hospital costs of out-of-hospital cardiac arrest patients treated in intensive care; a single centre evaluation using the national tariff-based system. BMJ Open. 2015;5:e005797. https://doi.org/10 1136/bmjopen-2014-005797.

4. Nolan JP, et al. Post-cardiac arrest syndrome: epidemiology, pathophysiology, treatment and prognostication. Resuscitation. 2008;79(3): 350-79.

5. Nolan JP, Morley PT, Vanden Hoek TL, Hickey RW, Kloeck WG, Billi J, Böttiger BW, Morley PT, Nolan JP, Okada K, Reyes C, Shuster M, Steen PA, Weil MH, Wenzel V, Hickey RW, Carli P, Vanden Hoek TL, Atkins D; International Liaison Committee on Resuscitation. Therapeutic hypothermia after cardiac arrest: an advisory statement by the advanced life support task force of the International Liaison Committee on Resuscitation. Circulation. 2003108 (1): 118-121. URL: https://www.ncbi.n/m.nih.gov/pubmed/12847056.

6. Kilgannon JH, Jones AE, Shapiro NI, Angelos MG, Milcarek B, Hunter K, Parrillo JE, Trzeciak S; Emergency Medicine Shock Research Network (EMShockNet) Investigators. Association between arterial hyperoxia following resuscitation from cardiac arrest and in-hospital mortality. JAMA 2010 ; 303 (21): 2165-2171. https://www.ncbi.nlm.nih.gov/pubmed/ 20516417

7. Elmer J, Scutella M, Pullalarevu R, Wang B, Vaghasia N, Trzeciak S, RosarioRivera BL, Guyette FX, Rittenberger JC, Dezfulian C; Pittsburgh Post-Cardiac Arrest Service (PCAS). The association between hyperoxia and patient outcomes after cardiac arrest: analysis of a high-resolution database. Intensive Care Med.; 41 (1): 49-57. URL: https://www.ncbi.nlm.nih.gov/ pubmed/25472570

8. Stub D, Smith K, Bernard S, Nehme Z, Stephenson M, Bray JE, Cameron P, Barger B, Ellims AH, Taylor AJ, Meredith IT, Kaye DM; AVOID investigators. Air versus oxygen in ST-segment-elevation myocardial infarction. Circulation 2015 ; 131 (24): 2143-2150. doi: 10.1161/CIRCULATIONAHA.114.014494 https://www.ncbi.nlm.nih.gov/pubmed/26002889.

9. Nolan JP, Soar J, Cariou A, Cronberg T, Moulaert VR, Deakin CD, Bottiger BW Friberg H, Sunde K, Sandroni C; European Resuscitation Council; European Society of Intensive Care Medicine. European Resuscitation Council and European Society of Intensive Care Medicine 2015 guidelines for postresuscitation care. Intensive Care Med. 2015 ; 41 (12): 2039-2056. https:// www.ncbi.nlm.nih.gov/pubmed/26464394.

10. Sandroni C, Cariou A, Cavallaro F, Cronberg T, Friberg H, Hoedemaekers C, Horn J, Nolan JP, Rossetti AO, Soar J. Prognostication in comatose survivors of cardiac arrest: an advisory statement from the European Resuscitation Council and the European Society of Intensive Care Medicine. Intensive Care Med. 2014;40(12):1816-31. https://www.ncbi.nlm.nih.gov/pubmed/ 25398304

11. Golan E, Barrett K, Alali AS, et al. Predicting neurological outcome after targeted temperature management for cardiac arrest: systematic review and meta-analysis. Crit Care Med. 2014;42:1919-30.

12. Paul M, Bougouin W, Geri G, et al. Delayed awakening after cardiac arrest: prevalence and risk factors in the Parisian registry. Intensive Care Med. 2016; 42:1128-36.

13. Behrends M, Niemann CU, Larson MD. Infrared pupillometry to detect the light reflex during cardiopulmonary resuscitation: a case series. Resuscitation. 2012;83(10):1223-8.

14. Heimburger D, Durand $M$, et al. Quantitative pupillometry and transcranial Doppler measurements in patients treated with hypothermia after cardiac arrest. Resuscitation. 2016;103:88-93.

15. Selig C, Riegger $C$, et al. Bispectral index (BIS) and suppression ratio (SR) as an early predictor of unfavourable neurological outcome after cardiac arrest Resuscitation. 2014:85(2):221-6.

16. Nunes RR, Chaves IMM, de Alencar JCG, et al. Bispectral index and other processed parameters of electroencephalogram: an update. Rev Bras Anestesiol. 2012;62:105-17. doi: 10.1016/S0034-7094(12)70109-5.

17. Liberati A, Altman DG, Tetzlaff J, Mulcrow C, Gotzsche PC, Ioannidis JPA, et al. The PRISMA statement for reporting systematic reviews and metaanalyses of studies that evaluate health care interventions: explanation and elaboration. BMJ. 2009;339:b2700

18. Higgins J, Green S, editors. Cochrane handbook for systematic reviews of interventions version 5.1.0 (updated March 2011). 5.1.0 ed. The Cochrane Collaboration; 2011. http://training.cochrane.org/handbook.

19. Sampson M, McGowan J, Cogo E, et al. An evidence-based practice guideline for the peer review of electronic search strategies. J Clin Epidemiol. 2009;62(9):944-52.

20. Cochrane Handbook of Systematic Review of Interventions. Version 5.1.0; 12 2.1 The GRADE approach. http://training.cochrane.org/handbook. 
21. Cochrane Haematological Malignancies Group (CHMG). Data extraction form (CHMG) template; 2007. p. 13.

22. Sterne $J A C H J$, Reeves $B C$ on behalf of the development group for ACROBAT-NRSI: A Cochrane risk of bias assessment tool: for nonrandomised studies of interventions (ACROBAT-NRSI). In., Version 1.0.0 edn; 2014.

23. Cochrane risk of bias tool for randomized controlled trials. http://methods. cochrane.org/bias/assessing-risk-bias-included-studies.

24. Hunter JE, Schmidt FL. Methods of meta-analysis: correcting error and bias in research findings. Newbury Park: Sage; 1990.

25. Higgins JPT, Thompson S, Deeks JJ, Altman DG. Measuring inconsistency in meta-analyses. BMJ. 2003;327(7414):557-60.

Submit your next manuscript to BioMed Central and we will help you at every step:

- We accept pre-submission inquiries

- Our selector tool helps you to find the most relevant journal

- We provide round the clock customer support

- Convenient online submission

- Thorough peer review

- Inclusion in PubMed and all major indexing services

- Maximum visibility for your research

Submit your manuscript at www.biomedcentral.com/submit
Biomed Central 